\title{
Building on the essential foundation of physiology
}

\author{
Clement L. Ren MD, MBA, Benjamin Gaston MD
}

To the Editor,

The recently published editorial in Pediatric Pulmonology (B Gaston, et al. Pediatr Pulmonol, 2020, available online) entitled "A proposal for the addressing the needs of the pediatric pulmonary work force" outlines the critical importance of expanding the scope of scientific training during pediatric pulmonology fellowship. To complement this document, we would like to reiterate the central importance of having a strong foundation in classic pulmonary physiology knowledge, such as respiratory mechanics, gas exchange, ventilation/perfusion relationships, control of breathing, and exercise physiology. The careers of many venerable pioneers of pediatric pulmonology began with the study of pulmonary physiology. Indeed, the scientific work 7 of the 10 recipients of the American Thoracic Society's Founder's Award (given to individuals in recognition of their role in founding the discipline of pediatric pulmonology) focused on physiology.

Providers other than pediatric pulmonologists often manage pediatric respiratory disorders, such as wheezing, cough, and respiratory complications of prematurity, all of which are very common. However, pediatric pulmonologists can add unique value by applying principles of pulmonary physiology and lung development to the care and study of pediatric respiratory

This is the author's manuscript of the work published in final edited form as:

Ren, C. L., \& Gaston, B. (2020). Building on the essential foundation of physiology. Pediatric Pulmonology, 55(10), 2492-2492. https://doi.org/10.1002/ppul.24950 
disease. The need for this added value is not likely to change. In-depth training in physiology is foundational to the subspecialty, as it is for other pediatric subspecialties like as cardiology and nephrology. To apply all the scientific advances and knowledge gained in the last few yearsand anticipated in the future - training in classic physiology will need to be augmented with additional education in the cellular, molecular, and genetic basis of respiratory function and disease as outlined in the editorial. We would like to amplify the principle, mentioned in the editorial, that comprehensive training in physiology will always constitute a core competency for pediatric pulmonologists. Therefore, more time for training in science, rather than less, is needed during fellowship. We believe that the founders would agree. 